Research Article

\title{
Classification of Mental Stress Using CNN-LSTM Algorithms with Electrocardiogram Signals
}

\author{
Mingu Kang, Siho Shin, Jaehyo Jung $\mathbb{D}$, and Youn Tae Kim \\ AI Healthcare Research Center, Department of IT Fusion Technology, Chosun University, 309 Pilmun-daero Dong-gu, \\ Gwangju 61452, Republic of Korea \\ Correspondence should be addressed to Jaehyo Jung; jh.jung207@gmail.com and Youn Tae Kim; petruskim@chosun.ac.kr
}

Received 30 March 2021; Revised 30 April 2021; Accepted 21 May 2021; Published 7 June 2021

Academic Editor: G R Sinha

Copyright (C) 2021 Mingu Kang et al. This is an open access article distributed under the Creative Commons Attribution License, which permits unrestricted use, distribution, and reproduction in any medium, provided the original work is properly cited.

The mental stress faced by many people in modern society is a factor that causes various chronic diseases, such as depression, cancer, and cardiovascular disease, according to stress accumulation. Therefore, it is very important to regularly manage and monitor a person's stress. In this study, we propose an ensemble algorithm that can accurately determine mental stress states using a modified convolutional neural network (CNN)- long short-term memory (LSTM) architecture. When a person is exposed to stress, a displacement occurs in the electrocardiogram (ECG) signal. It is possible to classify stress signals by analyzing ECG signals and extracting specific parameters. To maximize the performance of the proposed stress classification algorithm, fast Fourier transform (FFT) and spectrograms were applied to preprocess ECG signals and produce signals in both the time and frequency domains to aid the training process. As the performance evaluation benchmarks of the stress classification model, confusion matrices, receiver operating characteristic (ROC) curves, and precision-recall (PR) curves were used, and the accuracy achieved by the proposed model was $98.3 \%$, which is an improvement of $14.7 \%$ compared to previous research results. Therefore, our model can help manage the mental health of people exposed to stress. In addition, if combined with various biosignals such as electromyogram (EMG) and photoplethysmography (PPG), it may have the potential for development in various healthcare systems, such as home training, sleep state analysis, and cardiovascular monitoring.

\section{Introduction}

Stress is a mental and physical reaction that a person may feel when they find themselves in a difficult and/or unfamiliar environment or situation. Excessive stress accumulation can cause chronic diseases such as high blood pressure, heart disease, and cancer and, in severe cases, can lead to death $[1,2]$. For this reason, stress observation is becoming increasingly important in modern society.

Studies measuring stress by using various biological signals such as electroencephalography (EEG), electromyogram (EMG), oxygen saturation, and pulse waves have been published [3-5]. However, these measurement methods require expensive and bulky systems to acquire data, are complicated and expensive to use, and require signal analysis by experts.
Existing studies using EEG signals have analyzed stress using support vector machines (SVMs), multilayer perceptrons (MLPs), and naïve Bayes (NB) and have obtained accuracies of $75 \%, 85.20 \%$, and $64.29 \%$, respectively [6-8]. However, because these studies used only 15 EEGs as training data, underfitting can occur. Furthermore, because an EEG produces a 7-channel signal, it involves a complex and time-consuming process to measure stress signals. Previous studies using EMG signals analyzed by SVM achieved an $85 \%$ accuracy. However, despite the same action being taken (the characteristic movement of the muscles), the magnitude of the signal amplitude varies from measurement to measurement, and noise in the signal makes it difficult to extract accurate feature points [9].

Studies that classify stress using an electrocardiogram (ECG) have been the most popular because the signal 
acquisition method is simpler than other methods and a clear waveform is acquired. Two studies achieved $89.21 \%$ and $84.4 \%$ accuracy using SVM $[10,11]$, but extracting feature points can be difficult because of noise and the time required to measure multichannel ECG signals and because preprocessing is not always accurate. Two different studies achieved $75 \%$ and $89 \%$ accuracy by considering the standard deviation of the R-R interval of the heart rate variability (HRV) signal $[12,13]$. Accurate stress classification is difficult because it takes more than $5 \mathrm{~min}$ to calculate the standard deviation of the R-R interval, and because the difference in parameter values is minimal. Furthermore, because the ECG waveform is not accurate in the frequency domain, it is difficult to extract feature points, making it difficult to directly evaluate or minimize the effect of noise generated by the human body.

In addition, certain research results have exhibited $63.97 \%$ and $82.7 \%$ accuracy using fuzzy c-means (FCM) clustering and convolutional neural network (CNN) $[14,15]$. These studies have difficulty classifying stress signals because the distance between the center point and the data is slight, and the scale of the training data is small, making it easier for the occurrence of underfitting.

Certain earlier study results have exhibited $87.39 \%$ and 90.19\% accuracy using CNNs and convolutional recurrent neural networks (CRNNs) $[16,17]$. In these studies, the hierarchical structure of the stress classifier is complex, and there is a considerable amount of noise; therefore, it is difficult to achieve a high-stress classification accuracy by detecting an incorrect $R$ peak value. Models based on long short-term memory (LSTM) achieved $88.13 \%$ accuracy [18]. However, owing to the high noise of the ECG signal, it is difficult to calculate the root mean square (RMS) of the R-R interval.

The aforementioned stress signal classification algorithm using the ECG signal has disadvantages such as underfitting, the calculation of a standard deviation for the R-R interval of a long-time HRV signal, and the detection of an incorrect $R$ peak value. To overcome these problems, we propose an ensemble model that accurately classifies mental stress by combining CNN and LSTM. The proposed model extracts the $R-S_{\text {peak }}$ feature point using the threshold value, converts it into a spectrogram, and classifies the stress signal using ECG signal analysis.

To improve the stress classification accuracy, batch normalization (BN), flatten layers, and fully connected layers were added. Subsequently, the accuracy of the stress classification model was improved by separately classifying ECG signals in the time domain and frequency domain. Confusion matrices, receiver operating characteristic (ROC) curves, and precision-recall (PR) curves were used to evaluate the performance of the stress classification model. In this study, we proposed an ensemble method to classify the mental stress of the CNN-LSTM model using ECG signals. The data of the ST Change Database and WESAD Database were trained, and more than $98 \%$ classification performance was achieved.

\section{Materials and Methods}

2.1. Subject. Figure 1 shows the procedure for classifying stress signals. In this study, we used the ST Change Database and WESAD Database, which provide ECG signals that were acquired in different stress environments. The ST Change Database contains ECG data that records physical stress and consists of 28 ECG signals obtained from 15 male subjects [19]. The WESAD database contains 30 ECG signals measured at the wrist and chest obtained from 15 subjects (12 men and 3 women) [20].

\subsection{Preprocessing and Feature Extraction.} Electrocardiography is the most common way to check health status by noninvasively checking the electrical status of the heart. When taking an electrocardiogram, noise is generated by several factors, which greatly reduces ECG classification accuracy [21]. To solve this problem, we used a low-pass filter and confirmed that $90.89 \%$ of the noise was eliminated using a low-pass filter with a sampling frequency of $360 \mathrm{~Hz}$ and a cutoff frequency of $150 \mathrm{~Hz}$.

Figure 2 shows the extracted $R-S_{\text {peak }}$ values from an ECG signal. By extracting these data under stress and without stress, the ECG can be accurately analyzed [22]. $R_{\text {peak }}$ and $S_{\text {peak }}$ were extracted from ECG signals after setting a threshold. $R_{\text {peak }}$ extracted the pole when the threshold value was greater than $0.2 \mathrm{mV}$ in one period of the signal and extracted the pole when the threshold value was less than $-0.54 \mathrm{mV}$ in one period.

In the under-stress state, the heart beats irregularly and quickly, the R-R interval of the ECG signal becomes narrow, and the $R-S_{\text {peak }}$ increases. On the other hand, in the unstressed state, the heart is relatively stable, the R-R interval widens, and the $R-S_{\text {peak }}$ decreases [23]. In each state, the average $R-S_{\text {peak }}$ without stress was found to be $1.47 \mathrm{mV}$, and under stress, it was $4.25 \mathrm{mV}$. Figure 3 shows the conversion of either signal (under stress or without stress) into a spectrogram.

2.3. CNN-LSTM Model Design. Figure 4 shows the architecture of the ensemble model proposed in this study. The classification layer consists of 14 levels.

Table 1 lists the structure of the layers comprising the ensemble model. First, $124 \times 124 \times 3$ image sequence data are input to the sequence input layer. Subsequently, the ECG image data are converted into an array form (vertical, horizontal, and channel) using a sequence folding layer and then transferred to the convolution layer.

The reason for using the sequence folding layer is so that the image sequence data can be converted into an array, arranged, and then transferred to the two-dimensional (2D) convolution layer. The first $2 \mathrm{D}$ convolution layer contains six filters of size $5 \times 5$.

Because of calculating the convolution layer using equation (1), the size of the output value is $124 \times 124 \times 6$. Equation (1) represents the calculation process for the 


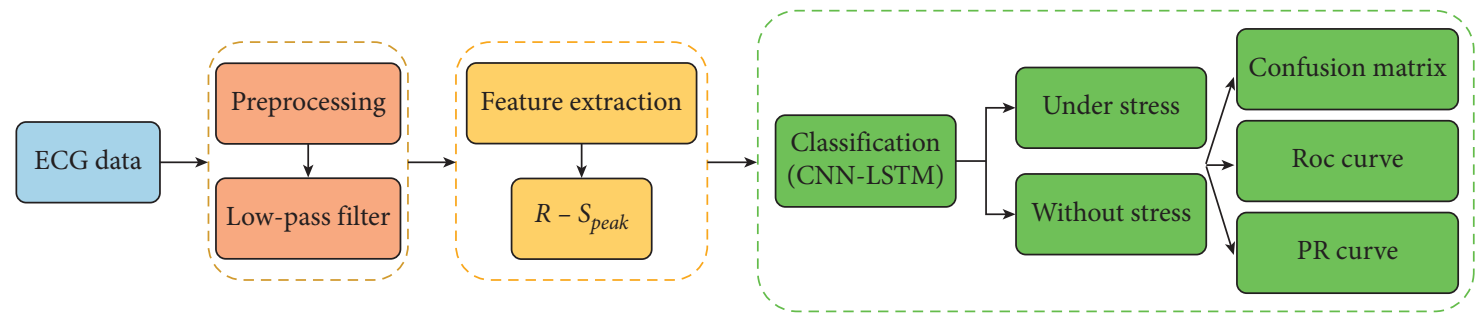

Figure 1: Procedure for classifying stress signals and validating the model.

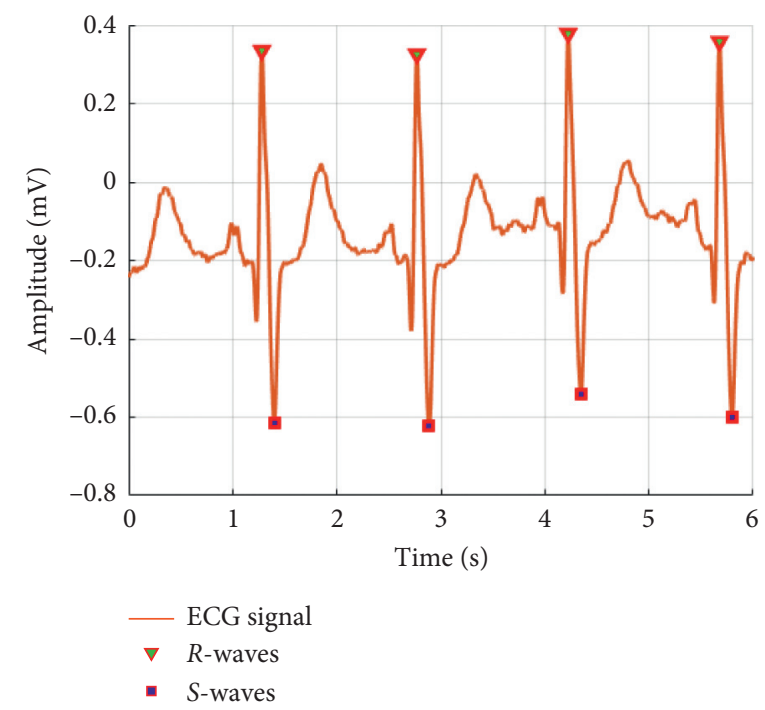

FIgURE 2: Feature point extraction by threshold.
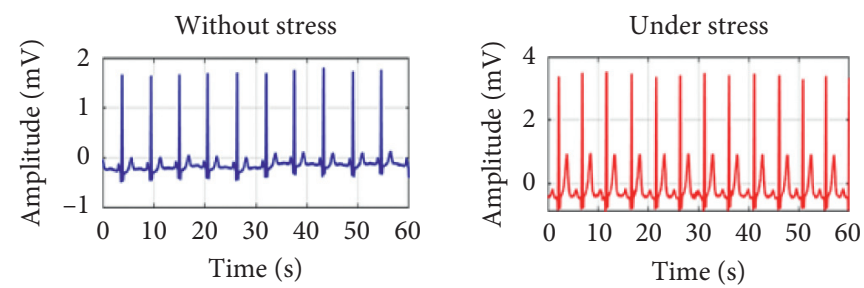

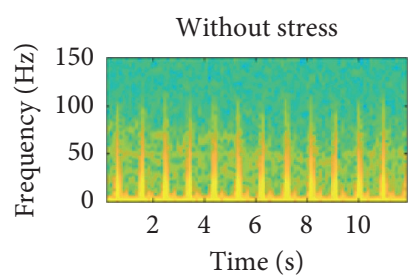

(a)

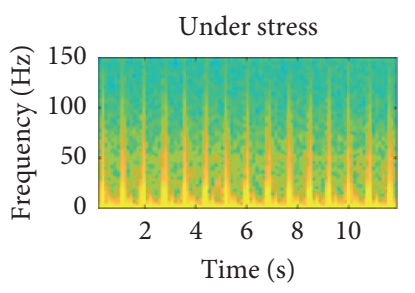

(b)

FIgURE 3: (a) Without stress ECG converted to a spectrogram. (b) Under stress ECG converted to a spectrogram.

convolution layer. When padding and stride are applied, and the size of the input data and filter is given, the output value can be calculated. $H$ and $W$ are the input data size, $F H$ (filter height) and $F W$ (filter weight) are filter size, $S$ is the stride, $P$ is padding, and $\mathrm{OH}$ (output height) and $O W$ (output weight) are output value sizes.

$$
(O H, O W)=\left(\frac{H+2 P-F H}{S}+1, \frac{W+2 P-F W}{S}+1\right)
$$

The output data are then connected to the batch normalization layer. After normalizing the size of the output 


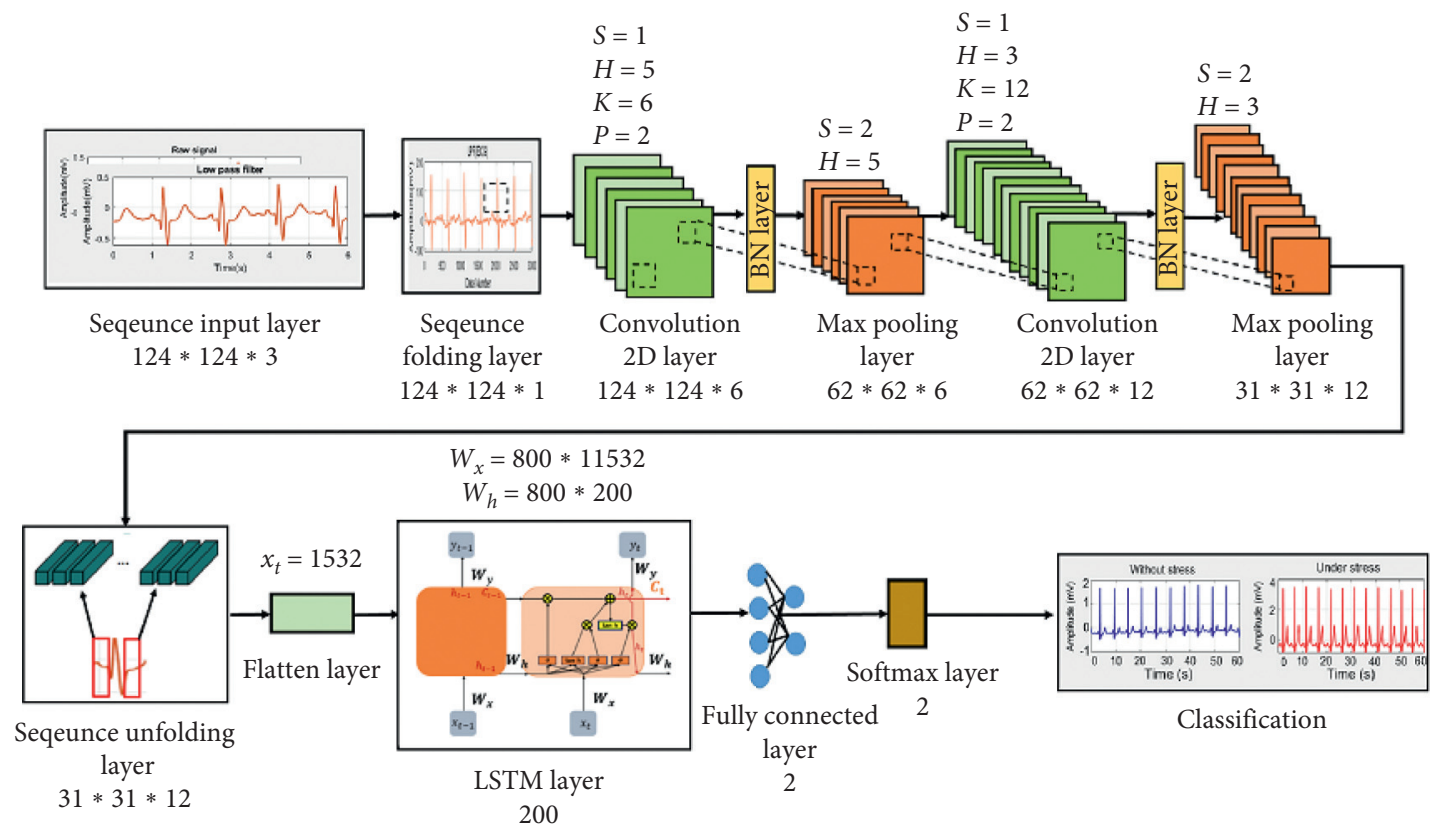

FIGURE 4: Classification architecture.

TABLE 1: Classification layers used to evaluate stress signals using CNN-LSTM.

\begin{tabular}{lcccc}
\hline Number & Layer & Activation & Weights & Bias \\
\hline 1 & Sequence input layer & $124 \times 124 \times 3$ & - & - \\
2 & Sequence folding layer & $124 \times 124 \times 1$ & $5 \times 5 \times 3 \times 6$ & - \\
3 & Convolution 2D layer & $124 \times 124 \times 6$ & - & - \\
4 & Batch normalization layer & $124 \times 124 \times 6$ & $3 \times 3 \times 6 \times 12$ & - \\
5 & Max pooling layer & $62 \times 62 \times 6$ & - & - \\
6 & Convolution 2D layer & $62 \times 62 \times 12$ & - & - \\
7 & Batch normalization layer & $62 \times 62 \times 12$ & - & - \\
8 & Max pooling layer & $31 \times 31 \times 12$ & - & - \\
9 & Sequence unfolding layer & $31 \times 31 \times 12$ & Input: $800 \times 11532$ recurrent: $800 \times 200$ \\
10 & Flatten layer & 11532 & $2 \times 200$ & - \\
11 & LSTM layer & 200 & - & - \\
12 & Fully connected layer & $2 \times 1$ & - \\
13 & Softmax layer & 2 & - & - \\
\hline
\end{tabular}

data to $124 \times 124 \times 6$ in the batch normalization layer, it was connected to the max pooling layer. According to equation (2), the size of the output data is determined by dividing the row and column size by the pooling size.

$$
(\mathrm{ORs}, \mathrm{OCs})=\left(\frac{H}{P}, \frac{W}{P}\right) .
$$

Its output is fed to a batch normalization layer and then to a max pooling layer. The max pooling layer is a $2 \times 2$ filter with a stride of 2 . As a result, the original data are reduced to a size of $62 \times 62 \times 6$. The second $2 \mathrm{D}$ convolution layer contains 12 filters of size $3 \times 3$. As a result, the data are further reduced to a size of $31 \times 31 \times 12$. Normalization is then performed and the data are passed to the LSTM layer. To transfer the size of the output data to the LSTM layer, normalization was performed using a sequence unfolding layer, and feature vectors were obtained using a flattening layer (or flattened layer).

The flattening layer has the advantage of not affecting the parameter by converting the output of the extracted feature map into a $1 \mathrm{D}$ array, which allows reconstructing the feature maps as the input to the LSTM [24]. At this time, the input is transmitted through the hidden layer of the LSTM.

A weight value of $800 \times 11532$ at the input layer is applied to equations (3)-(7), which represents the computational process of the LSTM layer, to extract the feature value. The LSTM layer consists of input gates $\left(i_{t}, g_{t}\right)$, forget gates $\left(f_{t}\right)$, and output gates $\left(O_{t}\right)$. The LSTM layer is composed of an input gate $\left(i_{t}, g_{t}\right)$, forgetting gate $\left(f_{t}\right)$, and output gate $\left(O_{t}\right)$. In each gate, a weight value is multiplied according to an input vector $\left(x_{t}\right)$, a hidden state $\left(h_{t-1}\right)$, and a cell state $\left(C_{t}\right)$ using the sigmoid and Tanh functions, and then a feature value is extracted. 


$$
\begin{aligned}
& i_{t}=\sigma\left(W_{x} x_{t}+W_{h i} h_{t-1}+b_{i}\right), \\
& g_{t}=\tanh \left(W_{x g} x_{t}+W_{h g} h_{t-1}+b_{g}\right), \\
& f_{t}=\sigma\left(W_{x f} x_{t}+W_{h f} h_{t-1}+b_{f}\right), \\
& O_{t}=\sigma\left(W_{x o} x_{t}+W_{h o} h_{t-1}+b_{o}\right), \\
& C_{t}=f_{t} \circ C_{t-1}+i_{t} \circ g_{t} .
\end{aligned}
$$

Subsequently, it is applied to equation (8) to pass the feature value calculated at the output gate to the output layer. Equation (8) is the process of extracting a required feature value from several feature values calculated at the output gate. After extracting a feature value from -1 to 1 using the Tanh function, the feature value in the range calculated using the output gate is transferred to the output layer.

$$
h_{t}=O_{t} \circ \tanh \left(C_{t}\right) \text {. }
$$

The feature value extracted from the LSTM layer classifies the image using a fully connected layer of size two and calculates a probability value for the image classified by the softmax layer. Subsequently, image classification is performed using the feature values extracted earlier using the fully connected layer, and the probability value of the classified image is calculated using the softmax layer. Finally, in the classification step, the signal is classified as either under stress or without stress.

Figure 5 shows the components of the convolution 2D layer and LSTM layer to which equations (1)-(8) are applied. Equations (1) and (2) show the calculation process of the convolution 2D layer among the CNN models, and equations (3)-(7) show the process of outputting feature values using the weight values of the input gate, forgetting the gate, and output gate in the LSTM layer. Equation (8) transfers the feature values in the range from the output gate to the output layer.

We used the confusion matrix, receiver operating characteristic (ROC) curve, and precision-recall (PR) curve to evaluate the stress signal classification performance of the proposed ensemble model [25]. The confusion matrix is a matrix that allows one to evaluate how accurately the predicted value is compared to the actual observed value. We used ECG data from the ST Change Database (DB) and the WESAD DB. The total number of data points was 58 . However, with such a small amount of data, it is difficult to accurately evaluate the stress signal classification model. Therefore, to improve the accuracy of the classification model and better analyze its performance, the data were doubled by transforming the time domain data to frequency domain data using the fast Fourier transform (FFT), as indicated in Figure 6. After preprocessing, the performance of the ensemble model was evaluated using 58 time domain data and 58 frequency domain data.

\section{Experimental Results}

Table 2 shows the accuracy, sensitivity, specificity, precision, and negative predictive values obtained to evaluate the classification model's performance using formulas (9)-(13) [26-28]. Formula (1) defines accuracy and indicates the probability of accurately classifying all under stress and without stress conditions. In the formula, TP, TN, FP, and FN indicate true positive, true negative, false positive, and false negative, respectively. For the time and frequency domains, the accuracies of the stress classifier were $94.8 \%$ and $98.3 \%$, respectively.

$$
\text { Accuracy }=\frac{\mathrm{TP}+\mathrm{TN}}{\mathrm{TP}+\mathrm{TN}+\mathrm{FP}+\mathrm{FN}} .
$$

Sensitivity refers to the proportion of data correctly classified as without stress to all without stress data (actual observed data). In the time and frequency domains, the sensitivities of the stress classifier were $96.4 \%$ and $100 \%$, respectively.

$$
\text { Sensitivity }=\frac{\mathrm{TP}}{\mathrm{FN}+\mathrm{TP}} .
$$

Specificity is the proportion of data correctly classified as under stress among all under stress data (actual observed data). In the time and frequency domains, the sensitivities of the stress classifier were $96.4 \%$ and $100 \%$, respectively.

$$
\text { Specificity }=\frac{\mathrm{TN}}{\mathrm{TN}+\mathrm{FP}} \text {. }
$$

Precision is the ratio of the data correctly classified by the stress classification algorithm as without stress to the value of all data classified as without stress. In the time and frequency domains, the precision of the stress classifier was $93.1 \%$ and $96.6 \%$, respectively.

$$
\text { Precision }=\frac{\mathrm{TP}}{\mathrm{TP}+\mathrm{FP}} .
$$

The negative predictive value is the ratio of data classified correctly as under stress to the actual value without stress data. In the time and frequency domains, the negative predictive values of the stress classifier were $96.6 \%$ and $100 \%$, respectively.

$$
\text { Negative Predictive Value }=\frac{\mathrm{TN}}{\mathrm{TN}+\mathrm{FN}} .
$$

Figure 6 shows the results of the classification model's performance using a confusion matrix. The matrix on the left of Figure 7 uses the data converted to the time domain, and the matrix on the right is the result of using the data in the frequency domain. The highest classification accuracy of the proposed ensemble model was $98.3 \%$ for the frequency domain. In previous studies, the accuracy of the model was 83.6\% [29]. These results indicate that accuracy was improved by $14.7 \%$ using the proposed ensemble compared to previous results. 


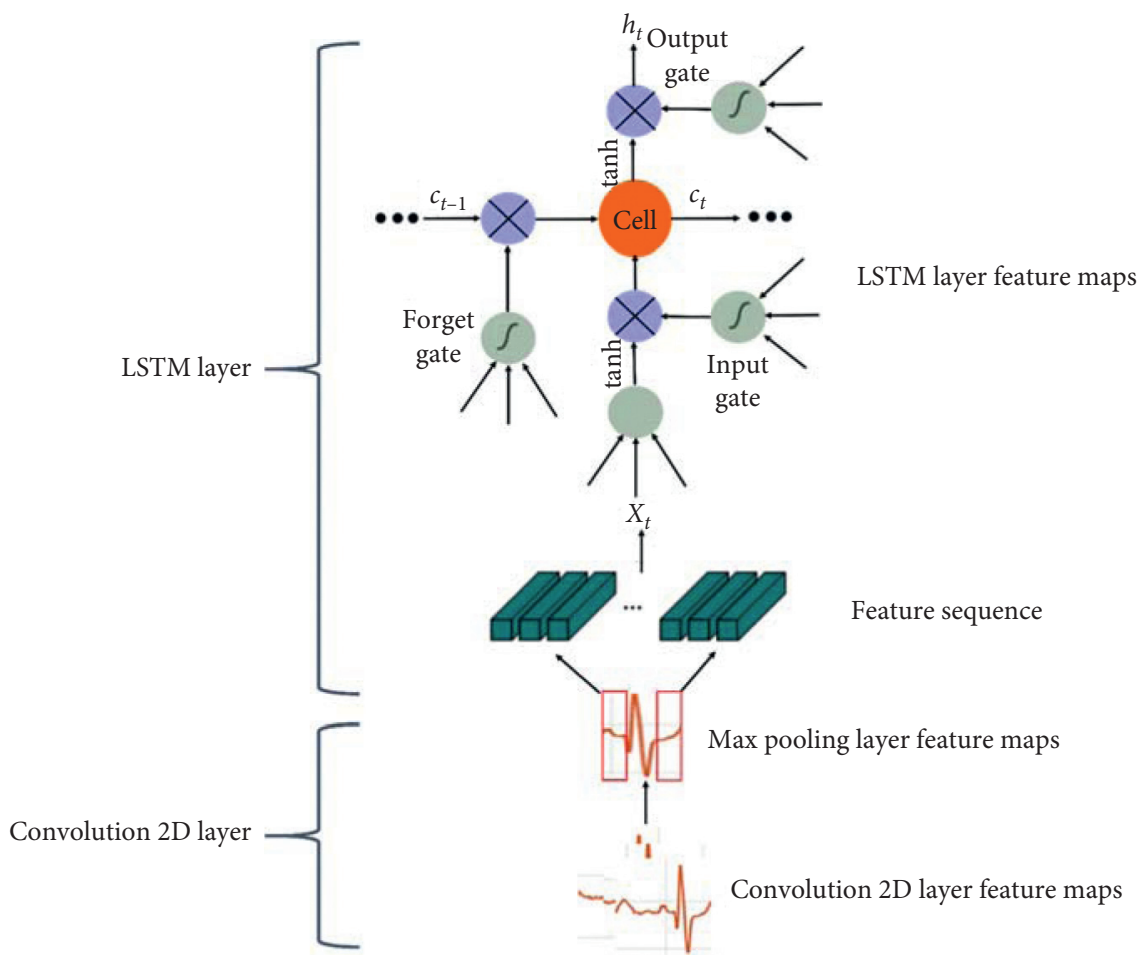

Figure 5: Components of the convolution 2D layer and the LSTM layer.

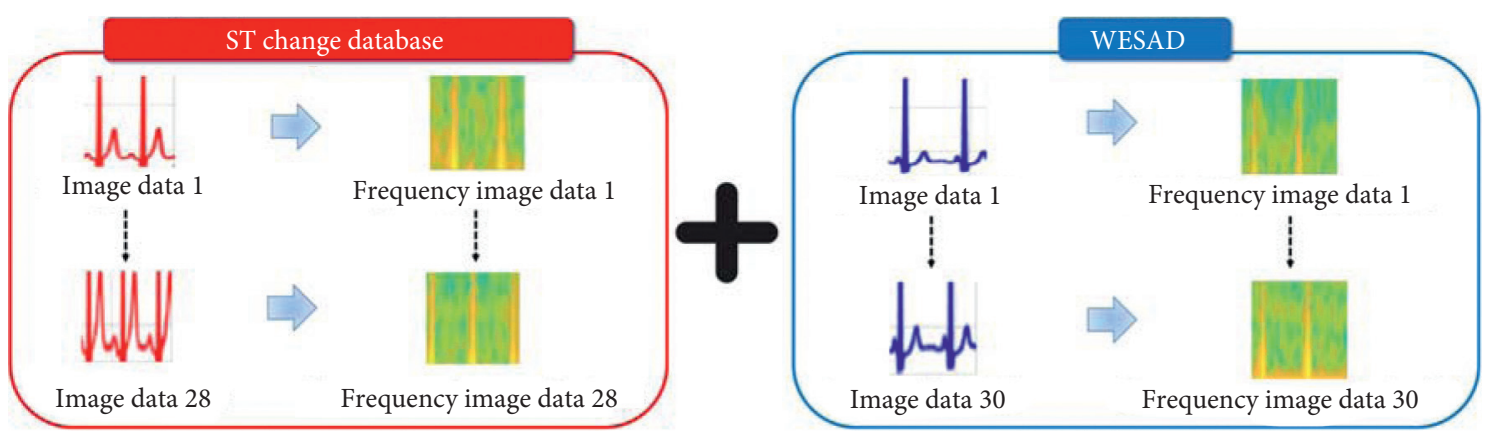

FIgURE 6: FFT transformation to increase input data quantity.

TABLE 2: Classification performance assessment of stress signals in time and frequency domains.

\begin{tabular}{|c|c|c|c|c|c|}
\hline \multicolumn{6}{|c|}{ Time domain } \\
\hline Stress & Precision & Sensitivity & Specificity & Negative predictive value & Accuracy \\
\hline Performance (\%) & $93.1 \%$ & $96.4 \%$ & $93.3 \%$ & $96.6 \%$ & $94.8 \%$ \\
\hline Error $(\%)$ & $6.9 \%$ & $3.6 \%$ & $6.7 \%$ & $3.4 \%$ & $5.2 \%$ \\
\hline \multicolumn{6}{|c|}{ Frequency domain } \\
\hline Stress & Precision & Sensitivity & Specificity & Negative predictive value & Accuracy \\
\hline Performance (\%) & $96.6 \%$ & $100 \%$ & $96.7 \%$ & $100 \%$ & $98.3 \%$ \\
\hline Error $(\%)$ & $3.4 \%$ & $0.0 \%$ & $3.3 \%$ & $0.0 \%$ & $1.7 \%$ \\
\hline
\end{tabular}

Figure 8 shows the classification performance according to the epochs for the time and frequency domains. The graph shows the mean squared error (MSE) according to the number of epochs. The time domain yielded the lowest MSE at 219 epochs (the validation curve shown), while the frequency domain yielded the lowest MSE at 223 epochs.
Figure 9 shows the ROC curves according to the epochs for the time and frequency domains of the ECG data. The ROC curve is a performance evaluation technique applicable to a binary classifier system that indicates how the performance of the classification model changes as the threshold changes [30]. The area under the curve (AUC) (the area under the ROC curve) is an index used to evaluate the 

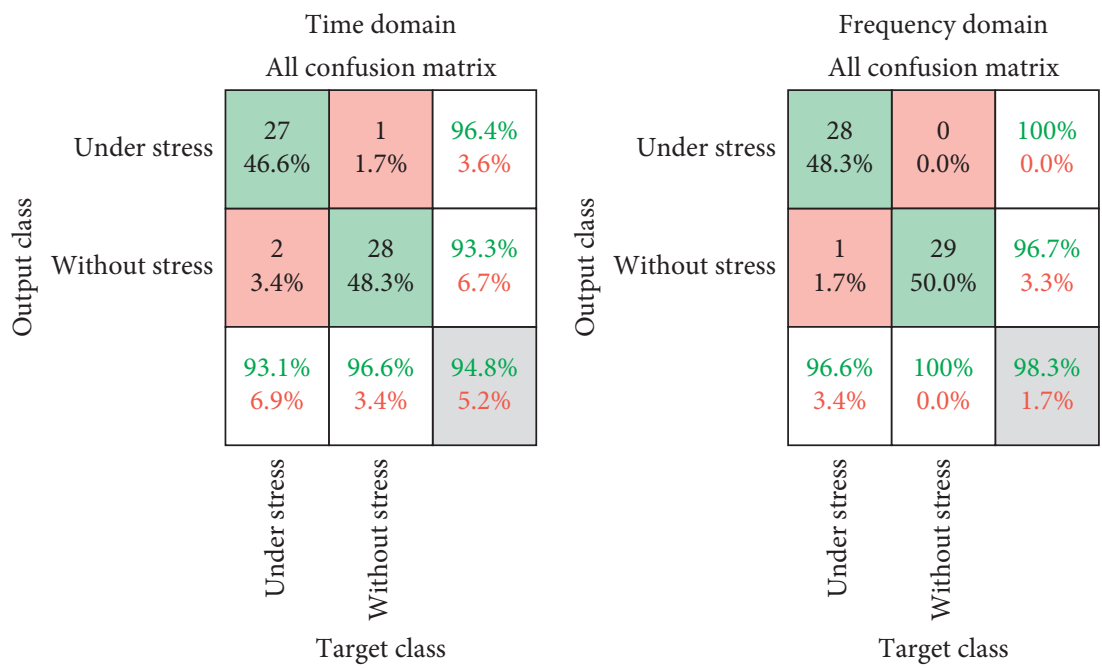

FIGURE 7: Classification performance evaluation of stress signals using a confusion matrix.
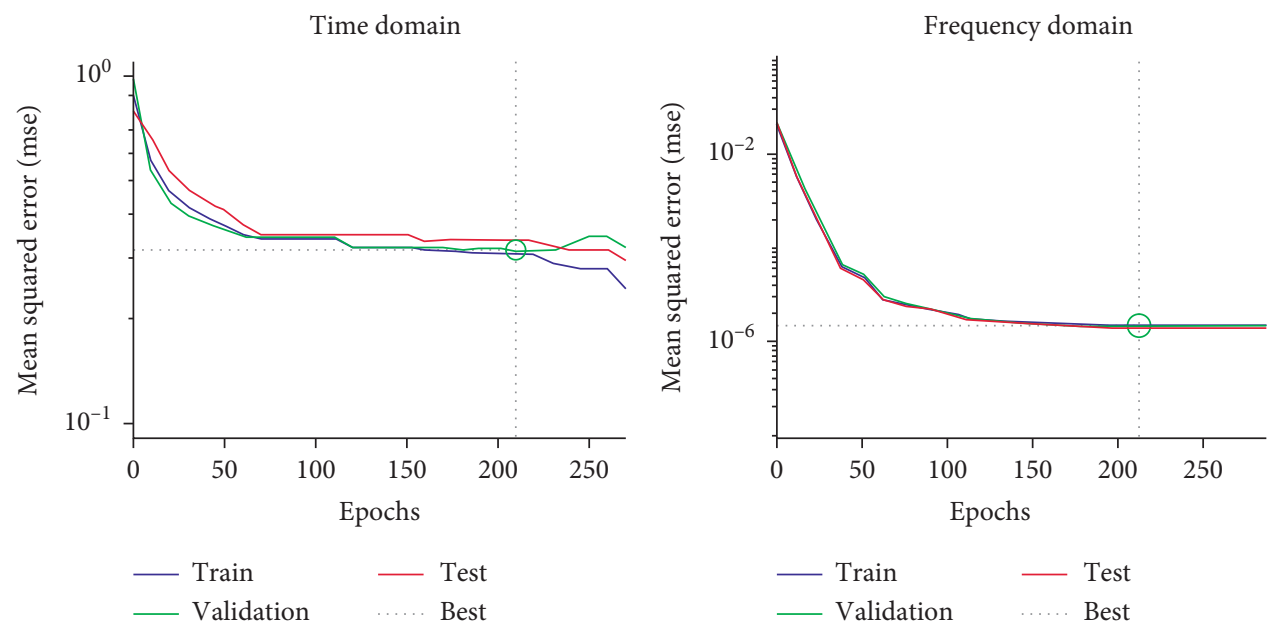

Figure 8: Classifier performance evaluation according to epoch.
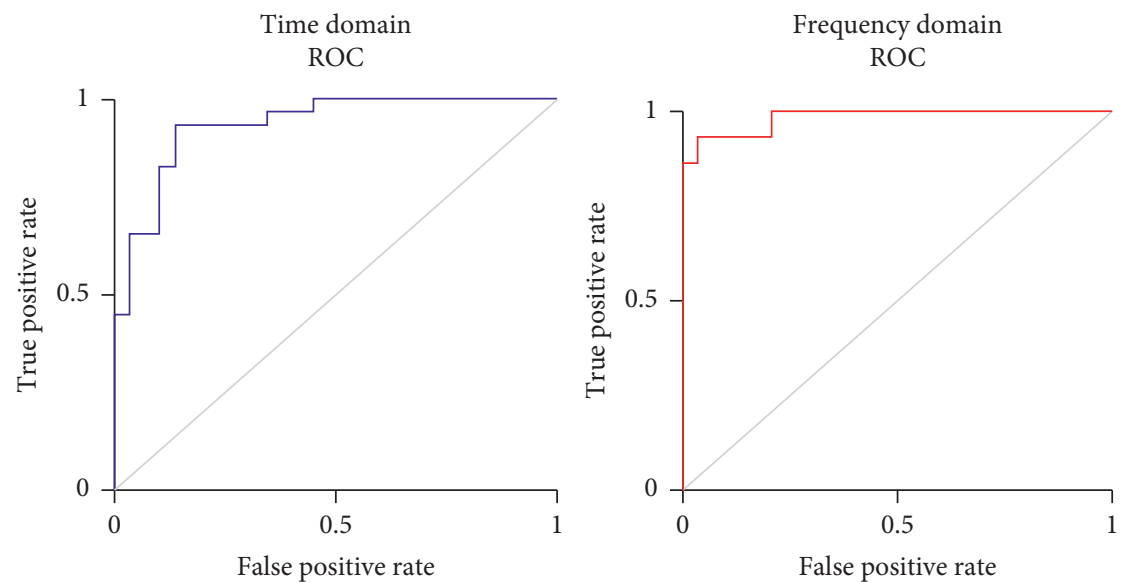

FIGURE 9: Stress classification performance evaluation using ROC curves. 
classification performance of different types of signals (stress signals in this study). When the AUC range falls between 0.9 and $1.0(90 \%-100 \%)$, the classification performance is excellent, and when the AUC range falls between 0.8 and 0.9 (80\%-90\%), the classifier's performance is low. In the time domain, the AUC of the ROC curve was $94.67 \%$, and it was $98.12 \%$ in the frequency domain. The AUC of a previous study was $85.7 \%$ [14], and it was confirmed that the ensemble proposed in this study represents a $12.42 \%$ improvement. The AUC value of the frequency domain was $3.45 \%$ higher than that of the time domain in our model indicating that the classification performance of the stress signal is better in the former.

Figure 10 shows the PR curves for the ECG data according to the epochs for the time and frequency domains. When considering the ROC curve, if the dataset is unbalanced, the shape of the curve is skewed to one side, and the classifier performance cannot be accurately evaluated [31]. The PR curve can be used to overcome the shortcomings of the ROC curve and shows the correlation between precision and recall. The average precision (AP) of the PR curve is an index that can be used to evaluate the classification performance of stress signals [32].

The $X$-axis represents the recall, and the $Y$-axis represents the precision. In the PR curve, the larger the AP is, the better the stress signal classification performance. The PR curve AP of the time domain was $93.8 \%$, and it was $97.6 \%$ for the frequency domain. The AP obtained using the PR curve in [32] was $84.2 \%$. Therefore, compared to the previously proposed stress signal classifier, the AP of the PR curve is improved by $13.4 \%$ using the proposed classifier. In addition, the AP value of the frequency domain was 3.8\% higher than that of the time domain in our model, indicating that the stress classification performance is better in the former.

In previous studies using the time domain or frequency domain of ECG data, the epochs were set to 10, and the batch size was set to 64 . As a result, the time domain and frequency domain accuracies were $83.6 \%$ and $74.5 \%$, respectively [33]. However, the architectures used are susceptible to overfitting, and the accuracies achieved after 10 epochs may reflect this problem. Figure 11 shows the accuracy of stress classification using the proposed CNN-LSTM. After setting the epochs to 20 and the batch size to 64 , the classification accuracies involving ECG stress signals in the time and frequency domains were measured. Under these settings, the time required for the time domain classification was $7 \mathrm{~min}$ $48 \mathrm{~s}$ and the verification accuracy was $94.13 \%$. The elapsed time for the frequency domain was $7 \mathrm{~min} 31 \mathrm{~s}$ and the verification accuracy was $98.26 \%$, which represents $10.53 \%$ and $23.76 \%$ improvements in accuracy compared to previous results [33].

For comparison purposes, we evaluated the stress classification performance of the CNN, LSTM, and CNN-LSTM models. First, stress signals were classified using CNN. After inputting the time series data values from the DBs into the image input layer, feature maps were extracted using convolutional, batch normalization, and max pooling layers. The stress was classified using a fully connected layer and a softmax layer under stress and without stress as the final classification. The classification accuracy of the stress signals using CNN was $88.35 \%$.

In addition, stress signals were classified using LSTM. LSTM is a type of recurrent neural network (RNN), which is an artificial neural network that recognizes patterns in data that can be represented as an array and is used for tasks such as text and gene signal analysis. After inputting the sequence data of the ECG DBs into the sequence input layer, the output was calculated using the LSTM layer (with the ReLU activation function). The signal was then classified as under stress or without stress using a fully connected layer. The classification accuracy of the stress signals using LSTM was $86.25 \%$.

Table 3 compares the stress classification accuracies of the CNN, LSTM, and CNN-LSTM models. We set the epochs to 20 and the batch size to 64 and then determined the elapsed time and accuracy. The results confirmed that the CNN-LSTM model was approximately 1 min faster than the CNN and LSTM models, and accuracy was improved by $9.91 \%$ and $12.01 \%$, respectively.

Figure 12 shows the AUC and AP curves for each model based on the ROC and PR results. The AUC of CNN-LSTM was $98.12 \%$, while those of CNN and LSTM were $87.5 \%$ and $84.3 \%$, respectively. Therefore, the AUC of the CNN-LSTM model was $10.62 \%$ and $13.82 \%$ higher than that of the CNN and LSTM models, respectively, confirming that its stress classification performance is better. The AP of CNN-LSTM was $97.6 \%$, and it was $88.2 \%$ and $86.02 \%$, respectively, for CNN and LSTM. The CNN-LSTM model achieved AP values that were $9.4 \%$ and $11.58 \%$ higher than the CNN and LSTM models, respectively, further confirming improved classification performance.

\section{Discussion}

In this study, to improve the performance of stress classification and prevent overfitting, an optimized ensemble model was developed by generating additional data using spectrograms and adding layers such as batch normalization, a flattening layer, and a fully connected layer. The performance of the classifier was evaluated using a confusion matrix, ROC, and other measures. By applying the average value of the $R-S_{\text {peak }}$ of the ECG signal, the characteristics of under-stress and without-stress signals are extracted to improve the stress classification accuracy. In the time domain, a precision of $93.1 \%$, a sensitivity of $96.4 \%$, and a specificity of $93.3 \%$ were achieved. In the frequency domain, a precision of $96.6 \%$, a sensitivity of $100 \%$, and a specificity of $96.7 \%$ were achieved. The CNN-LSTM achieved $94.8 \%$ accuracy for time domain signals and $98.3 \%$ accuracy for frequency domain signals. The best stress classification accuracy of the proposed CNN-LSTM algorithm is $98.3 \%$, which is approximately $14.7 \%$ higher than the best 

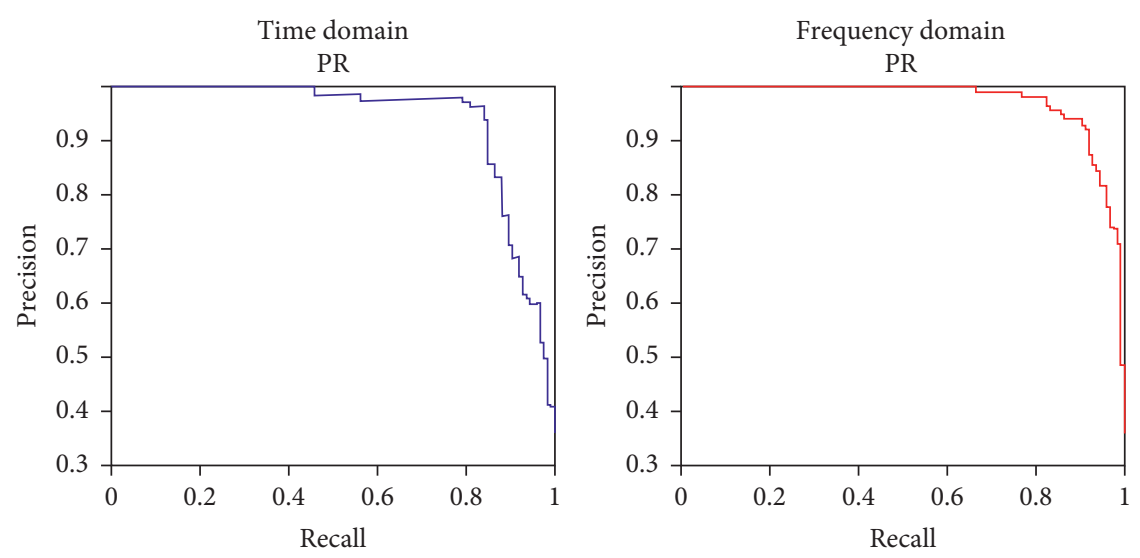

FIGURE 10: Evaluation of classification performance using PR curve.
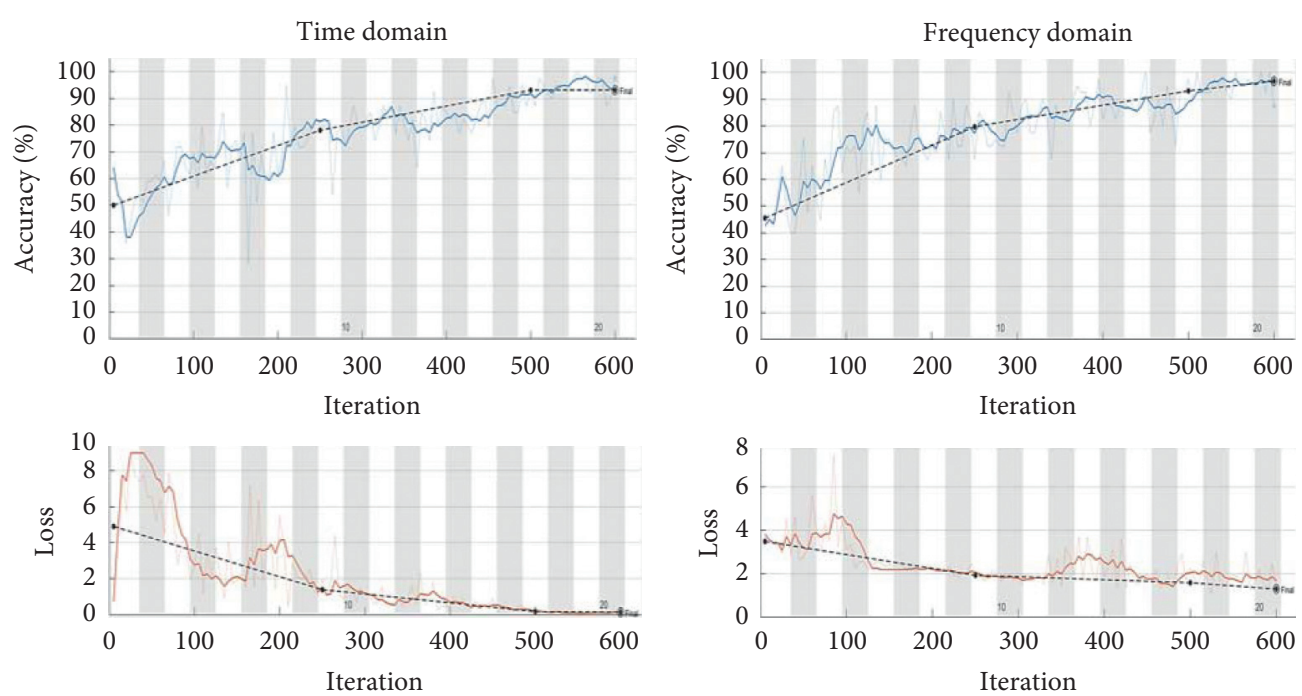

Figure 11: Time and frequency domain stress signal classification.

TABLE 3: Classification accuracy comparison of stress signals using CNN, LSTM, and CNN-LSTM.

\begin{tabular}{lccc}
\hline & CNN & LSTM & CNN-LSTM \\
\hline Elapsed time & $8 \min 32 \mathrm{~s}$ & $8 \mathrm{~min} 45 \mathrm{~s}$ & $7 \mathrm{~min} 31 \mathrm{~s}$ \\
Accuracy & $88.35 \%$ & $86.25 \%$ & $98.26 \%$ \\
\hline
\end{tabular}

accuracies reported in previous studies. The proposed stress classifier achieves optimal stress signal classification performance when the number of epochs is 219 in the time domain and 223 in the frequency domain. In addition, the model's performance was evaluated using ROC and PR curves. It was confirmed that improvements of $12.42 \%$ and $13.4 \%$, respectively, were obtained compared to previous study results. 

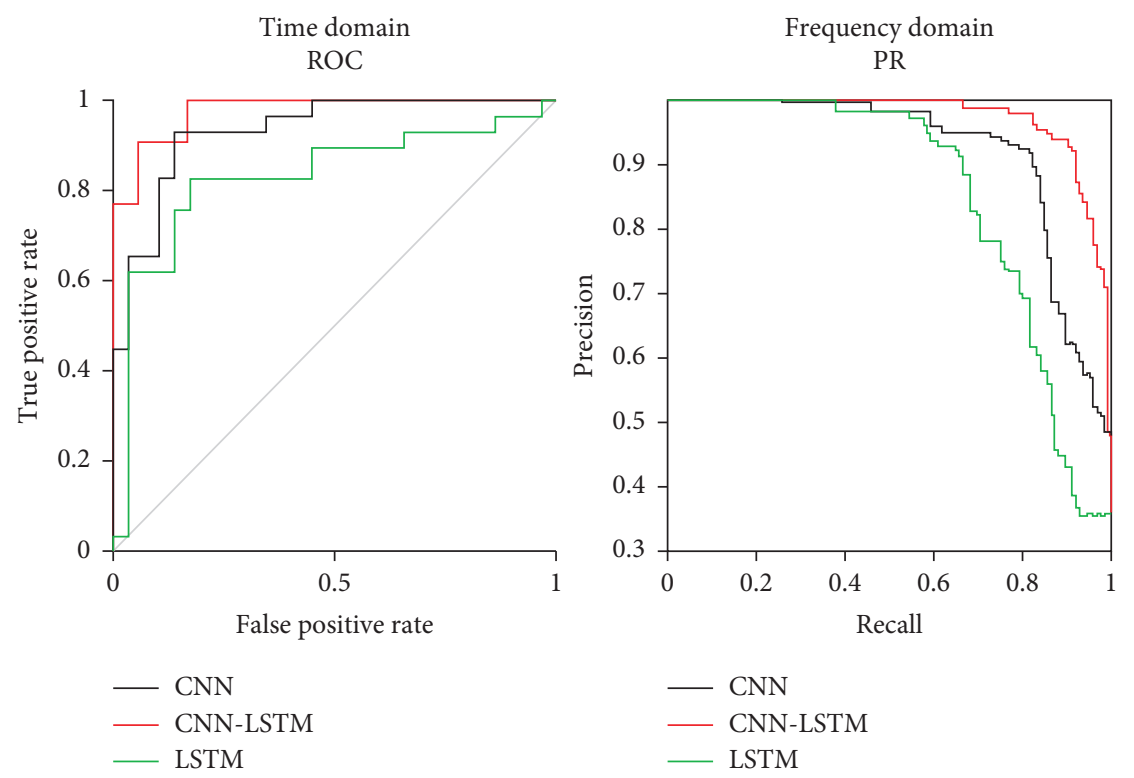

FIGURE 12: Evaluation of classification performance of stress signals using ROC and PR curves.

\section{Conclusions}

In this study, we proposed an improved ensemble model based on CNN-LSTM to accurately classify stress states. To prevent the overfitting of the algorithm and improve the accuracy of the classifier, ECG signals were classified separately in the time domain and frequency domain. The proposed ensemble model achieved a stress classification accuracy of $98.3 \%$. These results exhibit an approximate $14.7 \%$ improvement in accuracy compared to earlier studies that classify the existing under stress and without stress. In the future, we plan to improve the preprocessing method, such as a subtle noise removal of biological signals, and to improve accuracy by applying a wearable transform filter that will remove baseline fluctuations and noise using Fourier transforms. The stress classifier proposed by us is expected to be helpful in mental health management as it can quickly and accurately classify the stress experienced by modern people. It is also expected to assist in preventing various diseases such as depression, high blood pressure, and diabetes through periodic stress management.

\section{Data Availability}

The data are available at https://physionet.org/content/stdb/ 1.0.0/, https://archive.ics.uci.edu/ml/datasets/WESAD+\% 28Wearable+Stress+and+Affect+Detection $\% 29$.

\section{Conflicts of Interest}

The authors declare no conflicts of interest in this research.

\section{Acknowledgments}

This research was supported by the Healthcare AI Convergence R\&D Program through the National IT Industry Promotion Agency of Korea (NIPA) funded by the Ministry of Science and ICT (No. S1601-20-1041) and the Basic Science Research Program through the National Research Foundation of Korea (NRF) funded by the Ministry of Education (No. 2018R1A6A1A03015496).

\section{References}

[1] G. Giannakakis, D. Grigoriadis, K. Giannakaki, O. Simantiraki, A. Roniotis, and M. Tsiknakis, "Review on psychological stress detection using biosignals," IEEE Transactions on Affective Computing, vol. 2019, Article ID 2927337, 1 page, 2019.

[2] B. S. McEwen and E. Stellar, "Stress and the individual. Mechanisms leading to disease," Archives of Internal Medicine, vol. 153, no. 18, pp. 2093-2101, 1993.

[3] M. N. Dar, M. U. Akram, S. G. Khawaja, and A. N. Pujari, "CNN and LSTM-based emotion charting using physiological signals," Sensors, vol. 20, no. 16, pp. 4551-4552, 2020.

[4] Y. Jammes, J. G. Steinberg, O. Mambrini, F. Bregeon, and S. Delliaux, "Chronic fatigue syndrome: assessment of increased oxidative stress and altered muscle excitability in response to incremental exercise," Journal of Internal Medicine, vol. 257, no. 3, pp. 299-310, 2005.

[5] S. Elzeiny and M. Qaraqe, "Stress classification using photoplethysmogram-based spatial and frequency domain images," Sensors, vol. 17, pp. 312-313, 2020.

[6] A. Arsalan, "Classification of perceived human stress using physiological signals," in Proceedings of the 2019 41st Annual International Conference of the IEEE Engineering in Medicine and Biology Society (EMBC), pp. 1247-1250, IEEE, Berlin, Germany, July 2019.

[7] S. M. U Saeed, S. M. Anwar, H. Khalid, M. Majid, and U. Bagci, "EEG based classification of long-term stress using psychological labeling," Sensors, vol. 7, pp. 886-887, 2020.

[8] S. M. U. Saeed, S. M. Anwar, and M. Majid, "Psychological stress measurement using low cost single channel EEG headset," in Proceedings of the 2015 IEEE International Symposium on Signal Processing and Information Technology (ISSPIT), pp. 581-585, IEEE, Abu Dhabi, UAE, December 2015. 
[9] R. Luijcks, "Experimentally induced stress validated by EMG activity," PloS One, vol. 9, pp. 215-216, 2014.

[10] P. Zontone, A. Affanni, R. Bernardini et al., "Car driver's sympathetic reaction detection through electrodermal activity and electrocardiogram measurements," IEEE Transactions on Biomedical Engineering, vol. 67, no. 12, pp. 3413-3424, 2020.

[11] G. Giannakakis, K. Marias, and M. Tsiknakis, "A stress recognition system using HRV parameters and machine learning techniques," in Proceedings of the 2019 8th International Conference on Affective Computing and Intelligent Interaction Workshops and Demos (ACIIW), pp. 269-272, IEEE, Cambridge, UK, September 2019.

[12] S.-Y. Ye and D.-H. Kim, "Evaluation on the stress using HRV according to elapsed time of MRI noise," Journal of the Institute of Convergence Signal Processing, vol. 16, pp. 50-55, 2015.

[13] S.-Y. Dong, "Stress resilience measurement with heart-rate variability during mental and physical stress," in Proceedings of the 2018 40th Annual International Conference of the IEEE Engineering in Medicine and Biology Society (EMBC), pp. 5290-5293, IEEE, Honolulu, HI, USA, July 2018.

[14] H.-M. Cho, H. Park, S.-Y. Dong, and I. Youn, "Ambulatory and laboratory stress detection based on raw electrocardiogram signals using a convolutional neural network," Sensors, vol. 19, no. 20, pp. 4408-4410, 2019.

[15] B. Hwang, J. You, T. Vaessen, I. Myin-Germeys, C. Park, and B.-T. Zhang, "Deep ECGNet: an optimal deep learning framework for monitoring mental stress using ultra shortterm ECG signals," Telemedicine and E-Health, vol. 24, no. 10, pp. 753-772, 2018.

[16] B. S. Zheng, M. Murugappan, and S. Yaacob, "FCM clustering of emotional stress using ECG features," in Proceedings of the 2013 International Conference on Communication and Signal Processing, pp. 305-309, IEEE, Melmaruvathur, India, April 2013.

[17] J. He, K. Li, X. Liao, P. Zhang, and N. Jiang, "Real-time detection of acute cognitive stress using a convolutional neural network from electrocardiographic signal," IEEE Access, vol. 7, pp. 42710-42717, 2019.

[18] P. Zontone, A. Affanni, R. Bernardini, L. D. Linz, A. Piras, and R. Rinaldo, "Supervised learning techniques for stress detection in car drivers," Advances in Science, Technology and Engineering Systems Journal, vol. 5, no. 6, pp. 22-29, 2020.

[19] Z. Zhao, L. Yang, D. Chen, and Y. Luo, "A human ECG identification system based on ensemble empirical mode decomposition," Sensors, vol. 13, no. 5, pp. 6832-6864, 2013.

[20] P. Schmidt, "Introducing wesad, a multimodal dataset for wearable stress and affect detection," in Proceedings of the 20th ACM International Conference on Multimodal Interaction, pp. 400-408, Boulder, CO, USA, October 2018.

[21] V. Bhateja, S. Urooj, R. Mehrotra, R. Verma, A. Lay-Ekuakille, and V. D. Verma, "A composite wavelets and morphology approach for ECG noise filtering," in Proceedingds of the International Conference on Pattern Recognition and Machine Intelligence, pp. 361-366, Springer, Tezpur, India, December 2013.

[22] B. Porr and L. Howell, "R-peak detector stress test with a new noisy ECG database reveals significant performance differences amongst popular detectors," BioRxiv, vol. 4, pp. 722397-722399, 2019.

[23] R. Laszlo, H. J. Weig, S. Weretka, and J. Schreieck, "Narrow complex tachycardia with alternating R-R intervals during physical stress: double ventricular excitation," Indian Pacing and Electrophysiology Journal, vol. 8, pp. 129-132, 2008.
[24] C. I. Garcia, F. Grasso, A. Luchetta, M. C. Piccirilli, L. Paolucci, and G. Talluri, "A comparison of power quality disturbance detection and classification methods using CNN, LSTM and CNN-LSTM," Applied Sciences, vol. 10, no. 19, pp. 6755-6757, 2020.

[25] S. B. Shafiei, L. Zaeem, S. E. Ahmed, A. H. Ahmed, and A. G. Khurshid, "Identifying mental health status using deep neural network trained by visual metrics," Translational Psychiatry, vol. 10, pp. 1-8, 2020.

[26] T. Marwick, A.-M. D’hondt, T. Baudhuin et al., "Optimal use of dobutamine stress for the detection and evaluation of coronary artery disease: combination with echocardiography or scintigraphy, or both?" Journal of the American College of Cardiology, vol. 22, no. 1, pp. 159-167, 1993.

[27] D. Chicco, N. Tötsch, and G. Jurman, "The Matthews correlation coefficient (MCC) is more reliable than balanced accuracy, bookmaker informedness, and markedness in twoclass confusion matrix evaluation," BioData Mining, vol. 14, pp. 1-22, 2021.

[28] M. Ohsaki, P. Wang, K. Matsuda, S. Katagiri, H. Watanabe, and A. Ralescu, "Confusion-matrix-based kernel logistic regression for imbalanced data classification," IEEE Transactions on Knowledge and Data Engineering, vol. 29, no. 9, pp. 1806-1819, 2017.

[29] K. Behfarnia and F. Khademi, "A comprehensive study on the concrete compressive strength estimation using artificial neural network and adaptive neuro-fuzzy inference system," Iran University of Science \& Technology, vol. 7, pp. 71-80, 2017.

[30] D. J. Vining and G. W. Gladish, "Receiver operating characteristic curves: a basic understanding," Radiographics, vol. 12, no. 6, pp. 1147-1154, 1992.

[31] M. Mounir, Acoustic Event Detection: Feature, Evaluation and Dataset Design, pp. 1-171, Ku Leuven, Leuven, Belgium, 2020.

[32] S. Kumar, "StressNet: detecting stress in thermal videos," in Proceedings of the IEEE/CVF Winter Conference on Applications of Computer Vision, pp. 999-1009, Waikola, HI, USA, April 2021.

[33] Z. Ahmad and M. K. Naimul, "Multi-level stress assessment using multi-domain fusion of ECG signal," in Proceedings of the 2020 42nd Annual International Conference of the IEEE Engineering in Medicine \& Biology Society (EMBC), pp. 4518-4521, IEEE, Montreal, CA, USA, July 2020. 